\title{
Estudio del potencial energético undimotriz del departamento de Córdoba a través de un dispositivo absorbedor puntual tipo on-shore
}

\section{Wave energy resource assessment at department of Córdoba through point absorber wave energy converter type on-shore}

DOI: http://doi.org/10.17981/ingecuc.16.1.2020.010

Artículo de Investigación Científica. Fecha de Recepción: 7/12/2018. Fecha de Aceptación: 24/09/2019

\author{
Yahir González Dória \\ Universidad de Córdoba. Montería (Colombia) \\ yahirgonzalez@correo.unicordoba.edu.co \\ Manuel Herrera Medina \\ Universidad de Córdoba. Montería (Colombia) \\ malejandroherrera@correo.unicordoba.edu.co

\section{César Ávila Díaz} \\ Universidad de Córdoba. Montería (Colombia) \\ ci.avila.diaz@gmail.com
}

Para citar este artículo:

Y. González Dória, M. Herrera Medina, C. Ávila Díaz "Estudio del potencial energético undimotriz del departamento de Córdoba a través de un dispositivo absorbedor puntual tipo on-shore", INGE CUC, vol. 16, no. 1, pp. 141-155, 2020. DOI: http://doi.org/10.17981/ingecuc.16.1.2020.010

\section{Resumen}

Introducción- En Colombia la electrificación en zonas de difícil acceso sigue siendo un problema sin resolver, por tal razón las energías alternativas como la undimotriz presentan una posible solución para la obtención del recurso energético; sin embargo esta energía tiene poco desarrollo en el país.

Objetivo- El objetivo fue evaluar el potencial energético undimotriz del departamento de Córdoba.

Metodología- Se diseñó un dispositivo absorbedor puntual tipo on-shore capaz de transformar la energía potencial de las olas en electricidad para cuantificarla, validando su funcionamiento en un canal de oleaje que permite manipular variables como altura y periodo de ola.

Resultados- Los resultados obtenidos muestran que la potencia máxima producida por el dispositivo fue de $5.16 \mathrm{~W}$ con una altura de 0.3 metros y un periodo de 3.5 segundos.

Conclusiones- Se demostró que teóricamente existen 1058.4 $\mathrm{Wm}^{\wedge}(-1)$ de potencial energético undimotriz aprovechables en Córdoba, de los cuales con el dispositivo diseñado se podrían obtener cerca de $7.2 \mathrm{~W}$.

Palabras clave- Energía Undimotriz Colombia; Absorbedor puntual; Energías renovables

\begin{abstract}
Introduction- In Colombia, electrification in hard-to-reach areas remains an unsolved problem, for which reason alternative energies such as wave energy present a possible solution for obtaining the energy resource; However, this energy has little development in the country.

Objective- The objective was to evaluate the wave energy potential of the department of Córdoba.

Methodology- A specific on-shore absorbing device was designed, capable of transforming the potential energy of waves into electricity in order to quantify it, validating its operation in a wave channel that allows manipulation of variables such as wave height and period.

Results- The results obtained show that the maximum power produced by the device was $5.16 \mathrm{~W}$ with a height of 0.3 meters and a period of 3.5 seconds.

Conclusions- It was demonstrated that theoretically there are 1058.4 $\mathrm{Wm}^{\wedge}(-1)$ of usable wave energy potential in Córdoba, of which with the designed device one could obtain about $7.2 \mathrm{~W}$.

Keywords- Wave energy Colombia; Point absorber; Renewable energy
\end{abstract}




\section{INTRODUCCIÓN}

En Colombia las zonas no interconectadas eléctricamente con el sistema nacional representan aproximadamente el $32 \%$ del territorio nacional [1], allí los habitantes pueden abastecerse de energía eléctrica mediante plantas generadoras y conseguir iluminación recurriendo a la compra de velas, kerosene o gasolina para lámparas, pilas y baterías [2]. Teniendo en cuenta que los costos de la planta generadora, combustible, transporte y gastos adicionales asociados al funcionamiento de la misma son generalmente altos para comunidades que cuentan con pocos recursos económicos, se hace limitado el acceso al servicio eléctrico en dichos sitios, creando un ciclo negativo que les impide acceder completamente a servicios básicos de educación, salud y conservación de alimentos, este último de gran importancia dadas las altas temperaturas que se presentan en algunas regiones del país como el departamento de Córdoba; además de no contar con iluminación apropiada y constante en las horas nocturnas, lo que hace difícil las actividades cotidianas y fomenta una barrera significativa para el desarrollo [3].

Una posible solución para la problemática descrita sobre la producción de electricidad en zonas de difícil acceso es el uso de fuentes de energías no convencionales o renovables [4]. En Colombia a partir de la aprobación de la Ley 1715 de mayo del 2014 [L1] y el decreto 2143 de noviembre de 2015 [L2] referentes a la regulación de la integración de las energías renovables en el Sistema Eléctrico Nacional, donde se puede ver que el rol de estas energías ha ido creciendo poco a poco y que cada día son más importantes para el desarrollo del país. Dentro de las diferentes fuentes de energía renovable se encuentra una que ha sido poco estudiada en el país [5] y de manera casi nula en el departamento de Cordoba, está es la energía undimotriz, la cual utiliza como fuente las olas que se producen en la superficie del mar. A la fecha se han desarrollado una amplia gama de convertidores de energía undimotriz alrededor del mundo y se han patentado cerca de 1000 dispositivos [6], los cuales se pueden clasificar siguiendo varios criterios [7], [8]. Entre los principales diseños de convertidores se encuentran los de Columna de agua oscilante (OWC por sus siglas en ingles), los cuerpos oscilantes y los de tipo overtopping. Los dispositivos tipo on-shore son aquellos que se encuentran apoyados en la costa [9]. Los dispositivos tipo absorbedor puntual consisten en una boya con dimensiones horizontales pequeñas en comparación con la longitud de onda incidentes [10], la boya oscila con respecto a uno o más grados de libertad y la energía es absorbida amortiguando el movimiento de la boya.

En el mundo se han realizado diversos estudios sobre la energía undimotriz, en los cuales se ha estimado que el potencial de energía de las olas es aproximadamente de $2 \mathrm{TW}$ (1 teravatio= $10^{12}$ vatio) [11], [12] y de esa cantidad entre el $10 \%$ y $25 \%$ puede ser transformada en electricidad [13], no obstante a pesar de las ventajas de este tipo de energía renovable frente a otras, no presenta tanto desarrollo como el resto de energías [14], es por esto que su comercialización a gran escala es poca en todo el mundo. Pese a esto, la investigación en tecnologías y posibles sitios para su implementación se ha intensificado en los últimos años, dando como resultado el estudio de potencial en países como Italia [15], España [16], México [17], Chile [18], entre otros. Además de sitios específicos para probar nuevas tecnologías como por ejemplo España, que actualmente tiene tres sitios de prueba para la energía de las olas [19]: Biscay Marine Energy Platform (Bimep), la planta de energía de las olas de Mutriku que ahora es usada como sitio de prueba y PLOCAN en las islas Canarias, que incluye un área marítima de $23 \mathrm{~km}^{2}$ desde la costa hasta $600 \mathrm{~m}$ de profundidad. Durante el 2015 se instalaron y ensayaron dos aparatos de energía undimotriz en PLOCAN: el prototipo W1 desarrollado por la compañía española Wedge Global y el PENGUIN II prototipo desarrollado por la empresa WELLO instalado en septiembre de 2015.

En Colombia en el año 2006 se evaluaron los sitios en la geografía colombiana que debido a sus condiciones oceanográficas, cuentan con mayor potencial para la generación eficiente y económicamente competitiva de electricidad a partir de la energía contenida en los océanos [11], y luego, en el 2009 se analizaron la prefactibilidad para la generación de energía eléctrica mediante energía undimotriz en Tumaco y Nariño [24]. Más adelante, en el 2013 se estudia el aprovechamiento del recurso de energía de las olas en el mar caribe, específicamente en Isla Fuerte [25], teniendo en cuenta que esta zona del mar caribe no cuenta con la cantidad necesaria de instrumentos de medición y esto puede dificultar la evaluación adecuada del 
recurso, por lo cual se presenta una nueva metodología para estimar el potencial de energía de las olas en lugares en los cuales no se encuentre la documentación o los instrumentos necesarios para medir los parámetros de evaluación. A continuación, en el 2014 publican un artículo donde evalúan el potencial de energía de las olas en el mar Caribe [26], analizando información retrospectiva de 30 años, validándola con datos de boyas Colombianas y a través de tecnología satelital, enfocándose en el golfo de México y algunos lugares del mar Caribe que se encuentran en Colombia. Finalmente en el 2014 se publica un artículo que habla acerca de la generación undimotriz y la integración con la red, teniendo en cuenta el análisis de resonancia subsíncrona [27].

El presente artículo tuvo como objetivo principal evaluar el potencial energético undimotriz del departamento de Córdoba, con el fin de conocer la viabilidad de generación eléctrica a partir de ese recurso. Los pasos desarrollados para llevar a cabo el proyecto consistieron primero en identificar los valores típicos de altura y periodo de las olas en la zona costera del departamento de Córdoba, necesarios como parámetros de entrada para el diseño del dispositivo. A continuación se procedió a diseñar y construir un dispositivo absorbedor puntual tipo on-shore con base en la teoría hidrodinámica y normas ingenieriles; validando su funcionamiento en el canal de oleaje del laboratorio de hidráulica y mecánica de fluidos de la Universidad Nacional de Colombia (sede Medellín), donde se pueden variar alturas y periodos de ola. Finalmente se analizaron los datos obtenidos del dispositivo para relacionarlos con las condiciones propias del departamento.

\section{Desarrollo}

\section{A. Área de estudio}

El departamento de Córdoba se encuentra en la parte noroccidental de Colombia, región Caribe, localizado entre las coordenadas $09^{\circ} 25^{\prime}-07^{\circ} 15^{\prime} \mathrm{N}$ y $75^{\circ} 26^{\prime}-75^{\circ} 10^{\prime} \mathrm{W}$ (Fig. 1). Cuenta con $25058 \mathrm{~km}^{2}$ de territorio, de los cuales $124 \mathrm{~km}$ son costa en el mar caribe. Tiene un intervalo altitudinal entre 0-2200 metros, posee siete cuencas hidrográficas (Manso, Tigre, Sinú, Esmeralda, Verde, San Jorge y Canalete), numerosas ciénagas (289) y pantanos en una extensión total de 790 [29]. Además de contar con arroyos, quebradas y caños de aproximadamente $1.500 \mathrm{~km}$ de longitud [30].

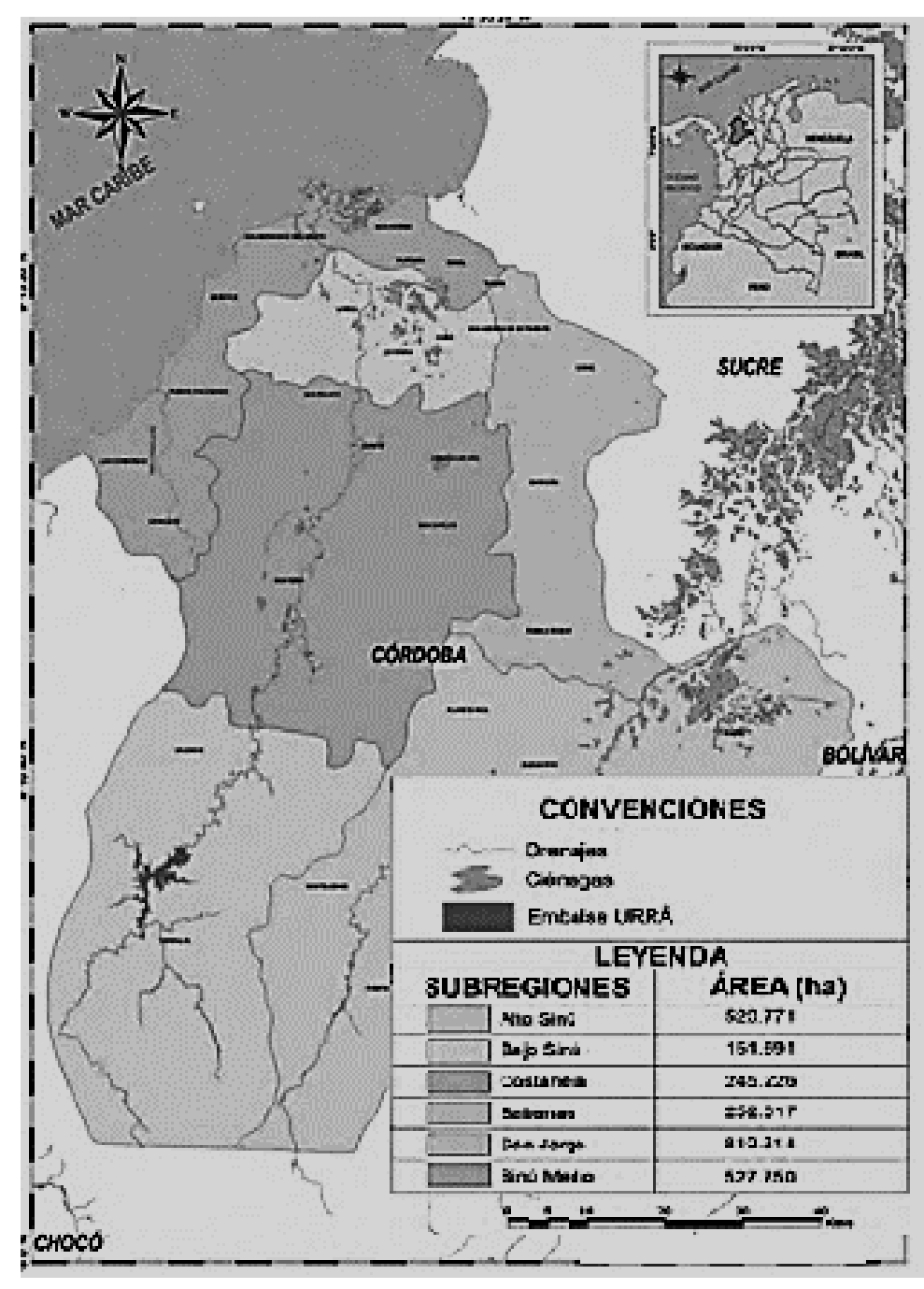

Fig. 1. Ubicación geográfica del departamento de Córdoba (Colombia). Fuente: [29]. 


\section{B. Fuentes de datos de oleaje}

En el año 2010 se presentó un trabajo en el cual se propone una metodología para estimar la cota de inundación en la zona costera del Caribe colombiano [31], el trabajo tiene un inciso en el cual para calcular el run up (cota que alcanza el oleaje al incidir sobre una estructura respecto el nivel del mar en reposo) se debe calcular el oleaje significativo. Para la región del Golfo de Urabá, la cual es la más cercana al departamento de Córdoba, se presentan según [31] valores del altura de ola que van desde la calma hasta valores máximos que alcanzan los $2 \mathrm{~m}$, con una media de $0.5 \mathrm{~m}$, y periodos que van desde $2 \mathrm{~s}$ hasta $29 \mathrm{~s}$, con una media de $6.7 \mathrm{~s}$.

La Dirección General Marítima-DIMAR cuenta con un sistema de Medición de Parámetros Oceanográficos y de Meteorología Marina-SMPOMM [34]. Este Sistema de medición permite obtener en tiempo real datos relacionados con las condiciones oceánicas y atmosféricas del territorio marítimo colombiano. La boya oceanográfica del SMPOMM que se encuentra más cerca al departamento de Córdoba es la Boya de Oleaje Turbo, la cual se encuentra aproximadamente a $50 \mathrm{~km}$ de la zona costera del departamento. Con la información obtenida por los elementos del Sistema SMPOMM, el Centro de Investigaciones Oceanográficas e Hidrográficas del Caribe-CIOH elabora los boletines Meteomarino del Caribe Colombiano mensuales [35]. Al revisar y analizar los datos de los boletines emitidos entre enero y diciembre de 2015, se comprobó como se muestra en la Tabla 1, que los datos obtenidos por [31] son cercanos a los obtenidos por las boyas y se concluyó que para efectos del diseño del dispositivo se tomaría una altura de ola promedio de $0.6 \mathrm{~m}$ y un periodo promedio de $6 \mathrm{~s}$.

Tabla 1. Altura y Periodo De ola mínimo En la zONA COSTERA DEL DEPARTAMENTO DE CÓRDOBA PARA EL AÑO 2015.

\begin{tabular}{|l|l|l|}
\hline \multicolumn{1}{|c|}{ Mes del año } & \multicolumn{1}{|c|}{ Altura de ola $(\mathrm{m})$} & Periodo de ola (s) \\
\hline Enero & 1 & 6 \\
Febrero & 0.6 & 4 \\
Marzo & 0.8 & 4 \\
Abril & 0.4 & 4 \\
Mayo & 0.4 & 3 \\
Junio & 0.4 & 3 \\
Julio & 0.4 & 6.5 \\
Agosto & 0.4 & 2 \\
Septiembre & 0.4 & 3.5 \\
Octubre & 0.2 & 2.5 \\
Noviembre & 0.4 & 4 \\
Diciembre & 0.6 & 5.5 \\
\hline Promedio anual & 0.5 & 4 \\
\hline
\end{tabular}

Fuente: Adaptada de [35].

\section{Metodología}

Para la realización de este trabajo se realizaron los pasos mostrados en la Fig. 2.

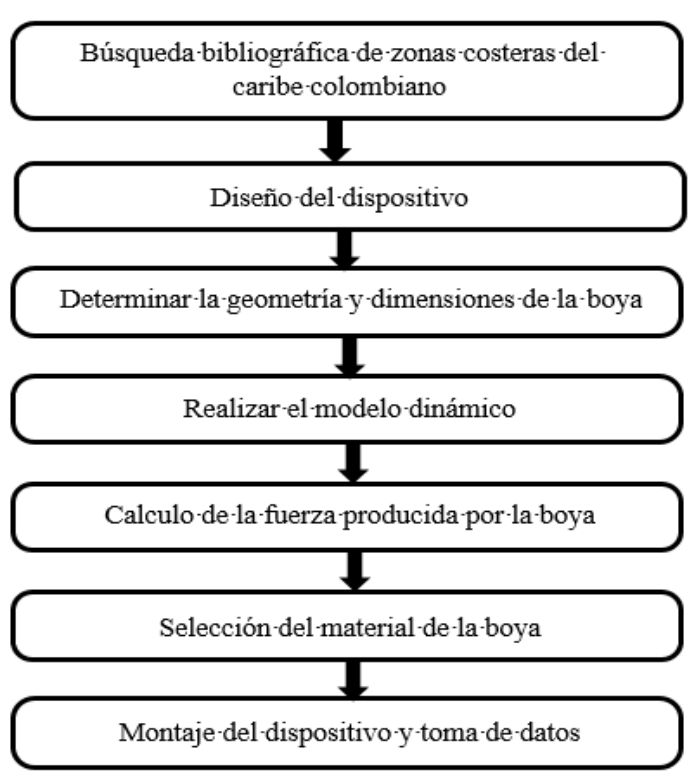

Fig. 2. Diagrama de flujo de la realización de la investigación Fuente: Autores. 


\section{A. Diseño del dispositivo convertidor de energía undimotriz}

Se diseñó un dispositivo convertidor de energía undimotriz tipo absorbedor puntual, el cual consta de una boya (1), un empalme (2), una cremallera de aluminio (3); un sistema de transmisión de potencia por piñones rectos $(4,7,8)$, ejes de aluminio $(5,9)$ soportados en rodamientos montados (10), un embrague unidireccional (6) y un generador eléctrico como se muestra en la Fig. 3 y Fig. 4. El principio de funcionamiento se basa en que la boya transmita la energía potencial de las olas hacia el sistema de transmisión de potencia, allí, el movimiento lineal se transforma en rotacional alternativo y luego de alternativo a rotacional unidireccional; finalmente el generador transforma la energía mecánica en eléctrica para su cuantificación a través de un multímetro conectado a un circuito.

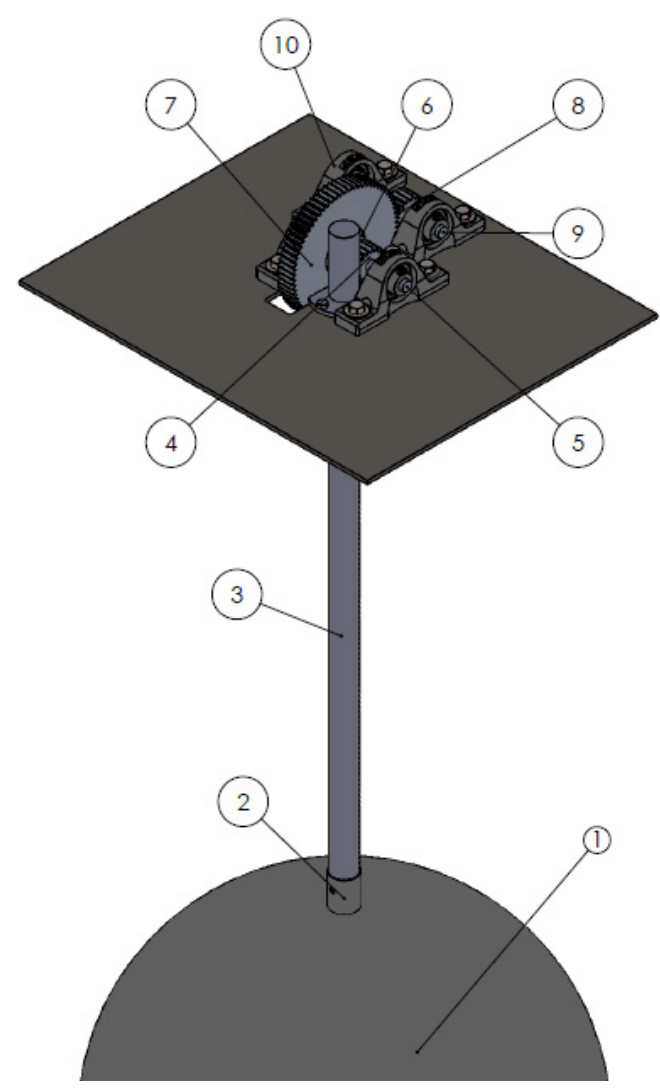

Fig. 3. Representación general del convertidor de energía undimotriz Fuente: Autores.

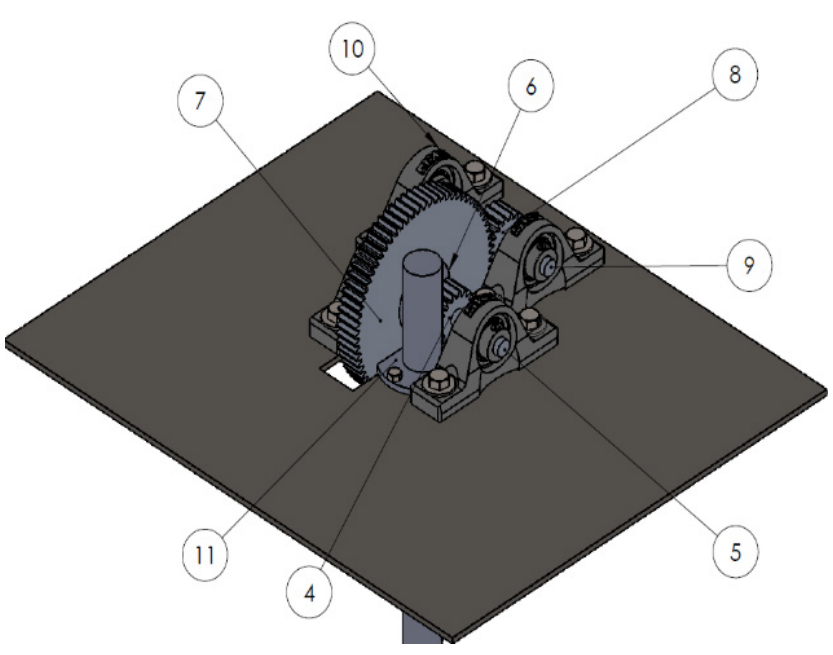

Fig. 4. Representación general del convertidor de energía undimotriz: vista ampliada Fuente: Autores.

\section{B. Geometría y dimensiones de la boya}

Según las conclusiones descritas por [36] los resultados de los análisis de elementos finitos para la carga, el dimensionamiento de los componentes y materiales elegidos por ellos, demostraron que la geometría de la boya esférica presenta los valores más bajos para las concentraciones de esfuerzos e induce los desplazamientos más bajos, dando lugar al mejor comportamiento estructural, con base en esto se eligió la geometría esférica para la boya; en el caso de la dimensión, se consideró que el ancho del canal de oleaje en el cual se realizaron las pruebas es de $1 \mathrm{~m}$, sin embargo por precaución al momento de ejecutar la validación se decidió elegir un diámetro de $0.6 \mathrm{~m}$. 


\section{Modelo dinámico del convertidor de energía undimotriz}

En la literatura científica diversos investigadores han utilizado la teoría hidrodinámica para modelar el comportamiento de los convertidores de energía undimotriz (en inglés Wave Energy Converter: WEC) en el mar, esta teoría descrita por [37]-[41] evalúa el movimiento de los WECs con base en la segunda ley de Newton. Lo anterior asumiendo que las amplitudes y oscilaciones son suficientemente pequeñas comparadas con la longitud de onda. Las componentes verticales de la fuerza total externa $f_{\text {ext }}$ actuando sobre la boya resultan de la suma de diversos componentes, los cuales se describirán más adelante, se tiene entonces por la teoría hidrodinámica (1):

$$
\sum f_{\text {ext }}=m_{b} \ddot{z}
$$

Donde $m_{b}$ y z̈ son respectivamente la masa de la boya y su correspondiente aceleración vertical. Para una boya esférica de radio $r$ su masa $m_{b}$ esta dada por (2):

$$
m_{b}=p_{b} V_{b} \Leftrightarrow m_{b}=p_{b} \frac{4}{3} \pi r^{3}
$$

Donde:

$p_{b}=$ densidad del material de la boya.

$V_{b}=$ volumen de la boya.

La ecuación (1) $\sum f_{\text {ext }}$ incluye el peso de la cremallera $w_{\text {crem }}$ que está encima de la boya, la fuerza de gravedad en función del desplazamiento como la describe [40] y la fuerza de las olas $f_{w}$, esta última se puede dividir en dos componentes hidrodinámicos actuando sobre la superficie de la boya: la fuerza de excitación $f_{e x c}$ debida a la incidencia de las olas actuando sobre la boya, asumiendo que esta se encuentra estacionaria, que para el presente diseño solamente se considera en modo tirante (en inglés heave) y la fuerza de radiación $f_{r}$ la cual corresponde a la fuerza experimentada por el cuerpo por su propio movimiento oscilatorio sobre el agua en calma o en ausencia de un campo de ola incidente. Por lo tanto una posible solución para (1) es (3):

$$
\begin{aligned}
& \left(m_{b}+m_{a}\right) \ddot{z}+B \dot{z}+C z= \\
& f_{w}+w_{\text {crem }}+f_{\text {gravedad }} \\
& \left(m_{b}+m_{a}\right) \ddot{z}+B \dot{z}+C z= \\
& f_{\text {exc }}+f_{r}+w_{\text {crem }}+f_{\text {gravedad }}
\end{aligned}
$$

Donde z̈ y z son respectivamente, la velocidad de la boya y el desplazamiento, $m_{a}$ es el coeficiente hidrodinámico de la masa agregada, que representa la inercia de un volumen de agua dado que rodea la boya, cuando esta se encuentra oscilando en modo tirante o heave [38]. B es el Coeficiente de Amortiguación de la Radiación (en inglés Radiation Damping Coefficient) el cual representa el amortiguamiento de la boya debido a la transferencia de energía de las olas radiadas lejos de la boya, cuando esta se encuentra en modo tirante o heave. C es el coeficiente de restauración o rigidez, el cual representa la posición instantánea de la boya con respecto a la superficie libre sin perturbaciones.

Para una boya esférica de radio $r$ la masa agregada $m_{a}$ puede ser hallada a través de (4) [42]:

$$
\begin{aligned}
m_{a} & =\frac{1}{2} p_{w} V_{b} \Leftrightarrow m_{a} \\
& =\frac{1}{2} p_{w} \frac{4}{3} \pi r^{3} \Leftrightarrow m_{a}=\frac{2}{3} p_{w} \pi r^{3}
\end{aligned}
$$

Donde $p_{w}$ corresponde a la densidad de agua de mar. 
En la ecuación (3) si B es considerado como un coeficiente de amortiguamiento lineal, Bż corresponde a la fuerza de amortiguación $f_{d}$ de acuerdo con [43], resolviendo por la ecuación de Morrison como muestra [44] se puede representar de la siguiente manera (5):

$$
f_{d}=B \dot{z} \Leftrightarrow f_{d}=\frac{1}{2} C_{d} p_{w} A_{\text {proy }} u^{2}
$$

Donde $C_{d}$ es el coeficiente de arrastre, $A_{\text {proy }}$ es el área proyectada normal de la boya normal al fluido y u es la velocidad del fluido no perturbado con respecto a la boya, la cual está dada según [44] por (6):

$$
u=\frac{\pi H}{T} e^{\left(\frac{2 \pi z}{\lambda}\right)} \cos (\omega t)
$$

Donde:

$\mathrm{H}=$ altura de la ola.

$\mathrm{T}=$ periodo de la ola.

$\lambda=$ longitud de onda de la ola.

$\omega=$ frecuencia angular de la ola.

En la ecuación (3), si C es considerado como un coeficiente lineal de restauración o de rigidez, $\mathrm{C}_{z}$ corresponde a la flotabilidad hidrostática denominada $f_{b}$ o fuerza boyante, la cual actúa sobre la boya debido a la posición instantánea de esta con respecto a la superficie libre de agua de mar, esta fuerza se puede expresar de la siguiente forma (7):

$$
f_{b}=C z \Leftrightarrow f_{b}=p_{w} g A z
$$

Donde:

$\mathrm{g}=$ aceleración debida a la gravedad.

$\mathrm{A}=$ área de sección transversal de la boya.

En la ecuación (3) la fuerza de radiación $f_{x}$ puede ser obtenida como se explica en [36], [38], asumiendo que la boya se comporta como una esfera de radio $r$ semi-sumergida en aguas profundas; de acuerdo a lo anterior la expresión para conocer puede escribirse (8):

$$
\begin{aligned}
f_{r} & =-R \dot{z} \Leftrightarrow f_{r} \\
& =-\left[\omega p_{w} \frac{2}{3} \pi r^{3}\left(\epsilon_{33}+i \mu_{33}\right)\right] \dot{z}
\end{aligned}
$$

Donde $\epsilon_{33}$ y $\mu_{33}$ son respectivamente los coeficientes no dimensionales en modo tirante o heave, de la resistencia a la radiación y la masa agregada. Los valores de estos parámetros pueden ser obtenidos de [38].

En la ecuación (3) la fuerza de excitación $f_{\text {exc }}$ cuando es aplicada a un cuerpo rígido que oscila por el oleaje depende de la elevación de la ola incidente. La relación que existe entre la elevación de la ola y la fuerza de excitación es según la teoría hidrodinámica no casual. La $f_{e x c}$ se puede describir a través de la siguiente expresión, la cual se basa en la relación que existe entre los coeficientes de radiación y los coeficientes de excitación, también llamada relación de Haskind según [38] (9):

$$
\begin{aligned}
f_{\text {exc }} & =\left|f_{\text {exc }}\right| \cos (\omega t) \Leftrightarrow f_{\text {exc }} \\
& =\left[k_{3} p_{w} g \frac{H}{2} \pi r^{2}\right] \cos (\omega t)
\end{aligned}
$$

Donde:

$k_{3}$ es el coeficiente de la fuerza de excitación en modo tirante o heave 
La ecuación (9) se utiliza para simplificar el modelo, considerando solamente ondas monocromáticas regulares [36]; el valor de $k_{3}$ puede ser encontrado en [38].

\section{Cálculos de la fuerza producida por la boya}

Para calcular la máxima fuerza producida por la boya se implementó una simulación del convertidor de energía undimotriz WEC siguiendo a [36], [45]. La simulación con dominio en el tiempo se ha desarrollado utilizando la herramienta Simulink del software Matlab \& Simulink Student Suite, el objetivo del programa es simular el comportamiento dinámico de los WEC debido a la acción de las olas del mar. Todas las fuerzas descritas en la sección anterior se agruparon en subsistemas individuales, tal como se puede apreciar en la Fig. 5.

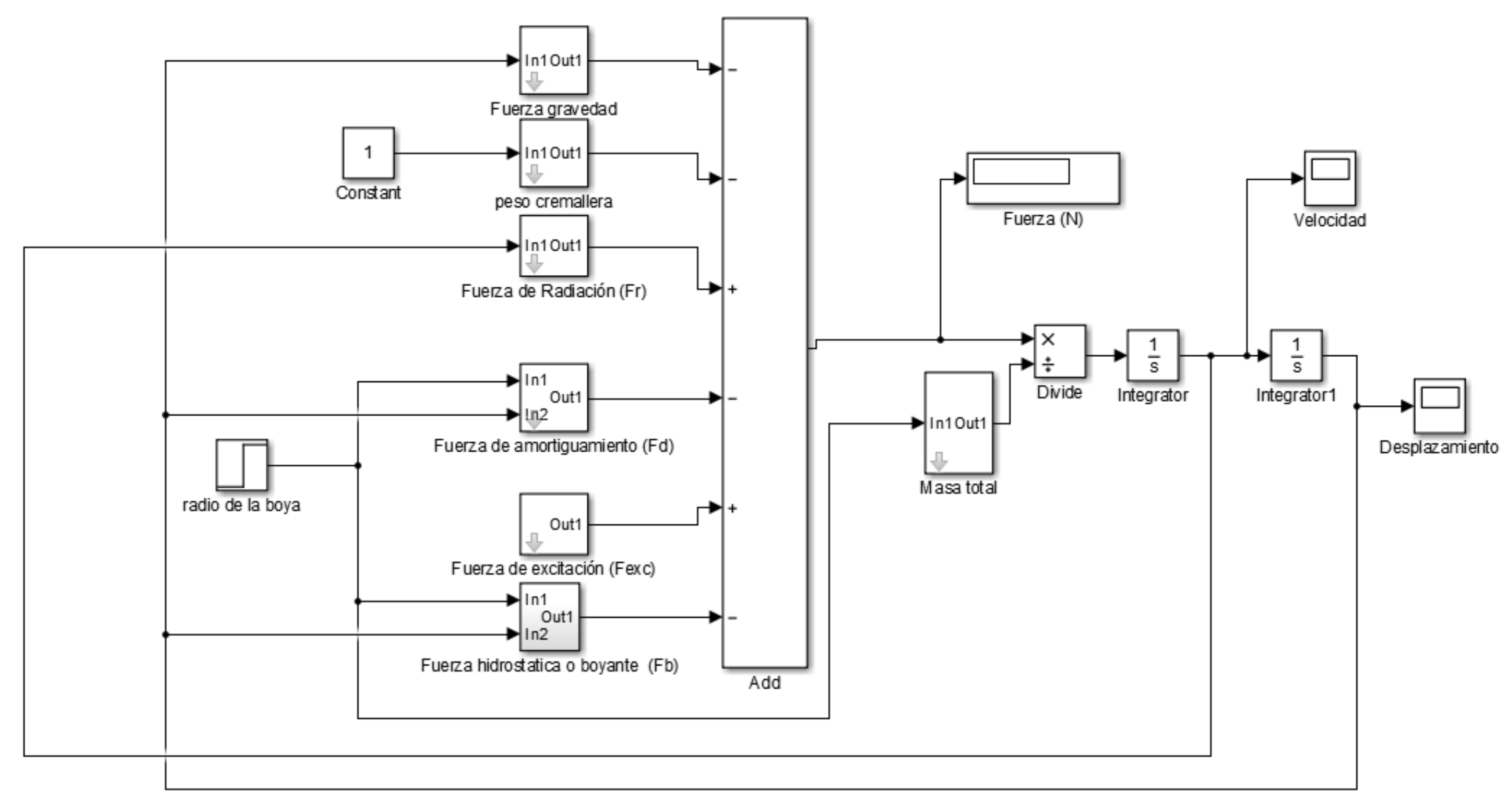

Fig. 5. Simulación de la dinámica del convertidor de energía undimotriz a través de la herramienta Simulink de Matlab Fuente: Autores.

Como resultado de la simulación se obtuvo que la fuerza que tendría que soportar la boya es de $500 \mathrm{~N}$ durante un intervalo de tiempo de $500 \mathrm{~s}$ (Fig. 6) utilizando una altura de ola de $0.6 \mathrm{~m}$ con un periodo de $6 \mathrm{~s}$ entre ola y ola. Este valor fue usado después para comprobar que el material elegido para la boya era el apropiado y como valor de entrada para el diseño del sistema de transmisión de potencia.

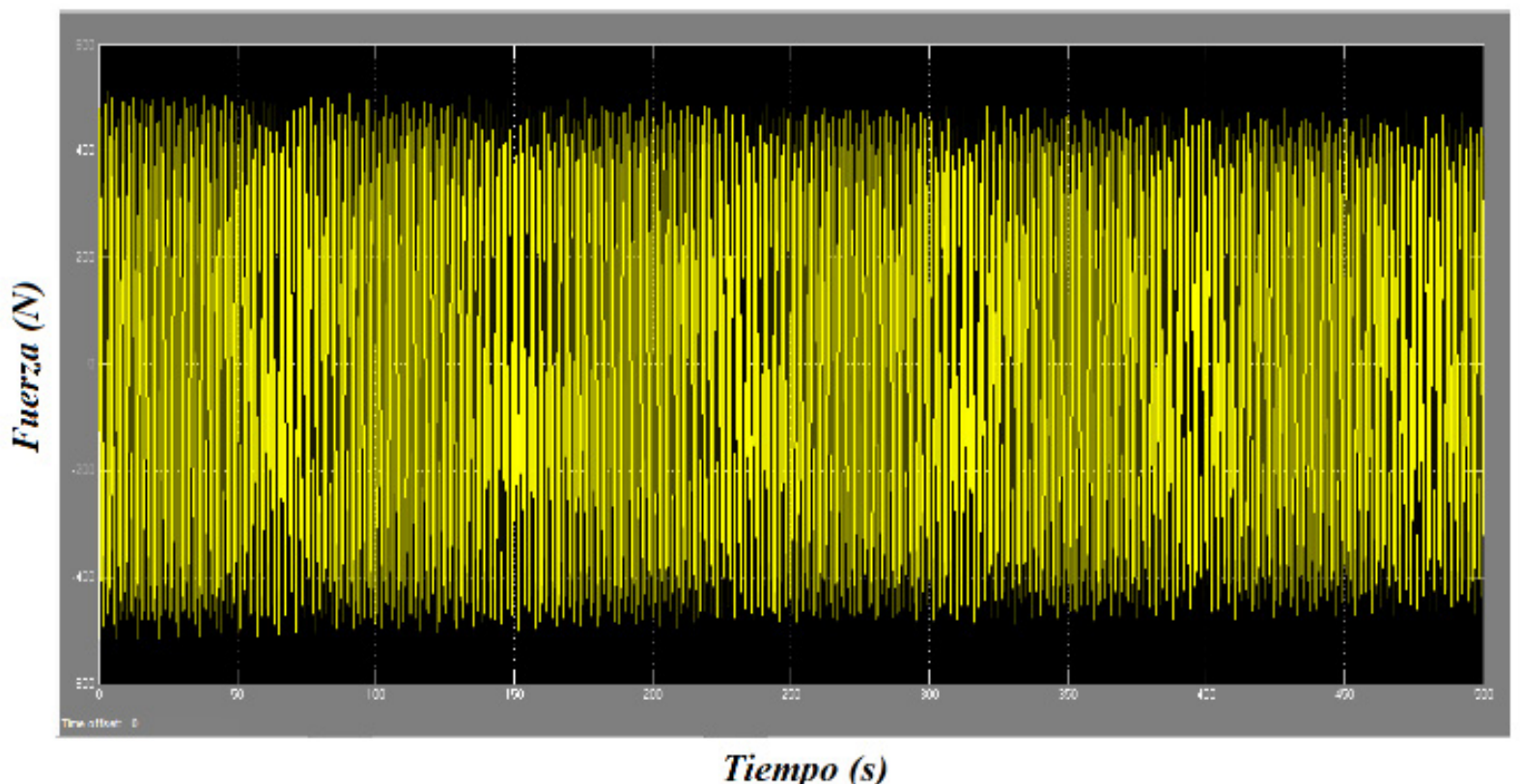

Fig. 6. Evolución de la fuerza total en el tiempo para alturas de ola de $0.6 \mathrm{~m}$ y periodos de $6 \mathrm{~s}$. Fuente: Autores. 


\section{Material para la construcción de la boya}

Se eligió fibra de vidrio como material para la construcción de la boya, debido a su baja densidad con respecto al agua, lo cual repercute en una alta flotabilidad, además de ser un proceso de manufactura económico en la ciudad de Montería con respecto al de los polímeros. Como se puede observar en la Fig. 7, en la boya no se generan esfuerzos que superen el esfuerzo al que resiste el material, y de igual manera se observa que los desplazamientos no superan los $0.003278 \mathrm{~mm}$, lo cual indica que la deformación de la boya es casi nula.
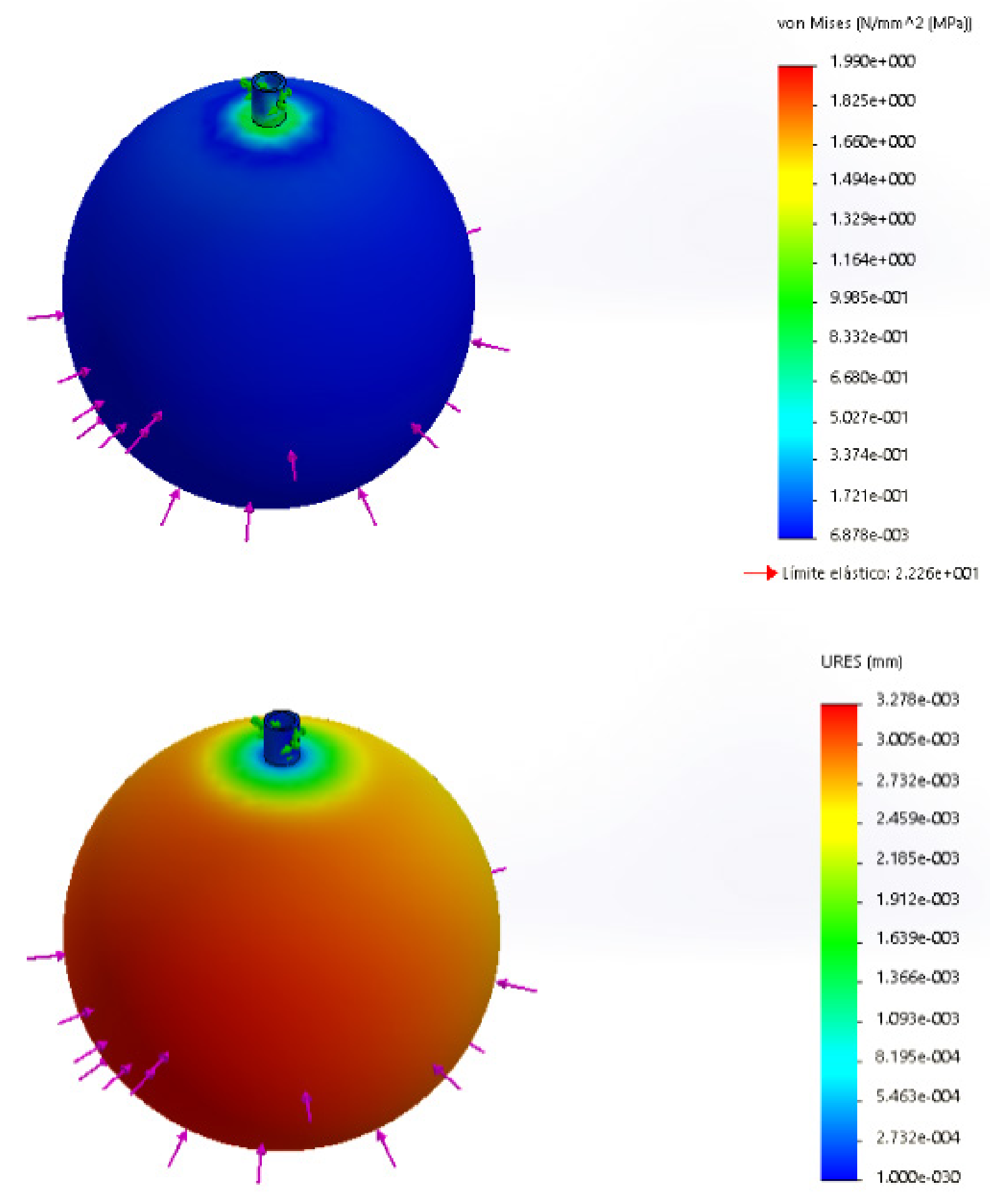

URES (mm)

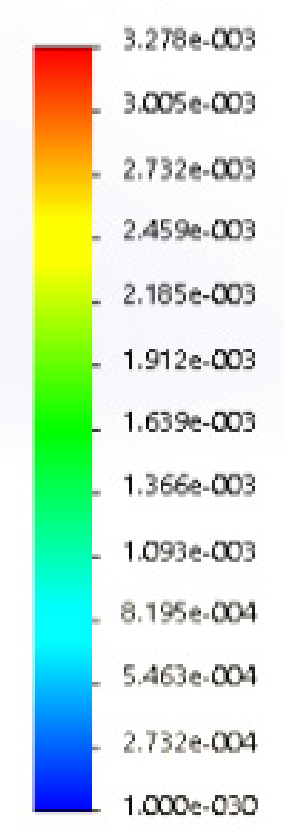

Fig. 7. Esfuerzos de Von Mises y desplazamientos para la boya recubierta en fibra de vidrio con radio 0.3 $\mathrm{m}$ considerando que se encuentre parcialmente sumergida. Fuente: Autores.

\section{Montaje del dispositivo y toma de datos}

El dispositivo se puso en marcha en el canal de oleaje del laboratorio de hidráulica y mecánica de fluidos de la Universidad Nacional de Colombia (sede Medellín). El canal cuenta con una longitud de $25 \mathrm{~m}, 1 \mathrm{~m}$ de ancho y $1 \mathrm{~m}$ de profundidad, puede producir olas de diferentes alturas desde $0.07 \mathrm{~m}$ hasta $0.3 \mathrm{~m}$, con periodos que van desde $0.5 \mathrm{~s}$ hasta $3.5 \mathrm{~s}$. El sistema de empuje puede reproducir oleaje monocromático con teoría del oleaje Stokes y oleaje espectral para los espectros teóricos Jonswap y Pierson-Moskowitz. Además cuenta con un sistema de disipación pasivo que absorbe $\sim 70 \%$ de la energía, apoyado por un sistema de corrientes que puede trabajar contra la ola o a favor de ella, para caudales máximos de hasta 90 litros por segundo. El montaje se hizo como se puede apreciar en la Fig. 8. 


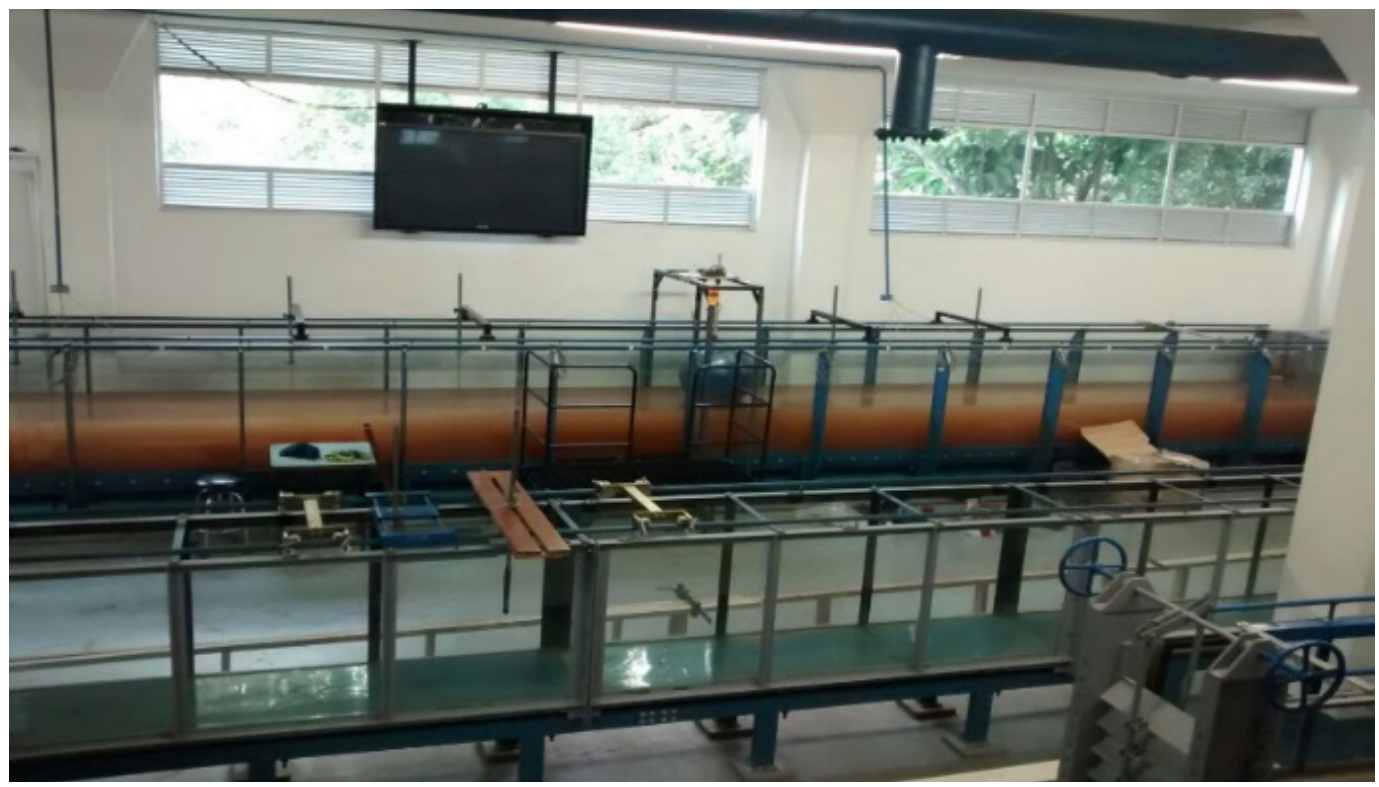

Fig. 8. Montaje del dispositivo en el canal de oleaje. Fuente: Autores.

Para la toma de datos se decidió utilizar un número representativo de 12 escenarios, los cuales se muestran en la Tabla 2, cada uno de ellos se ejecutó por un periodo de $60 \mathrm{~s}$. En cada uno de los escenarios se compararon las revoluciones teóricas calculadas según el desplazamiento de la cremallera, contra las reales medidas por medio de un tacómetro para verificar el funcionamiento del equipo.

TABla 2. Escenarios Elegidos PARA la toma DE DATOS.

\begin{tabular}{|l|l|l|}
\hline Escenario & Altura de ola $(\mathrm{m})$ & Periodo de ola $(\mathrm{s})$ \\
\hline 1 & 0.30 & 3.5 \\
2 & 0.30 & 2.5 \\
3 & 0.30 & 2.0 \\
4 & 0.25 & 3.5 \\
5 & 0.25 & 2.5 \\
6 & 0.25 & 2.0 \\
7 & 0.20 & 3.5 \\
8 & 0.20 & 2.5 \\
9 & 0.20 & 2.0 \\
10 & 0.15 & 3.5 \\
11 & 0.15 & 2.5 \\
12 & 0.15 & 2.0 \\
\hline
\end{tabular}

Fuente: Autores.

A continuación y teniendo en cuenta (10) que rige la potencia undimotriz, la cual es descrita en detalle por [12], [20], [23]; se calculó para cada uno de los escenarios de la Tabla 2 el potencial energético undimotriz teórico y se comparó con el potencial producido por el dispositivo; para calcular este último se multiplicó el voltaje por el amperaje medidos con el multímetro, teniendo en cuenta que estas mediciones se hicieron utilizando solo una bobina de las dos que posee el generador, esto quiere decir que los valores se duplicaron para el cálculo.

$$
P=\text { potencia }\left[\frac{\mathrm{kW}}{\mathrm{m}}\right]=0.49{\mathrm{H}_{\mathrm{s}}}^{2} \mathrm{~T}
$$

Luego conociendo los valores de potencia teórica y real, se determinó la eficiencia en cada uno de los escenarios de la Tabla 2 para analizar el comportamiento del convertidor.

Para finalizar y poder determinar cómo se comportaría el dispositivo en condiciones reales del departamento, se realizó una regresión lineal múltiple, esto para encontrar la ecuación que modela la potencia del dispositivo en función de la altura y el periodo de ola; para ello se utilizó el software estadístico R [46] en el cual primero se encontró el modelo de regresión de primer orden para los valores dados y luego se comprobó que esta ecuación tuviera los ajustes necesarios para que los valores de predicción fueran fiables, esto último se hizo por medio de test de validación de supuestos como son los de homogeneidad de varianza, independencia y normalidad. 


\section{RESUltAdOs}

En la Tabla 3 se muestran los datos de revoluciones obtenidos a través del análisis teórico del desplazamiento de la cremallera y los tomados por medio del tacómetro en cada uno de los escenarios; al analizarlos se puede observar que los escenarios 1, 4, 7 y 10 son los que más se aproximan, esto puede ser debido a que los cuatro tuvieron en común el periodo de ola de $3,5 \mathrm{~s}$, tiempo en el cual la boya alcanzaba a realizar un recorrido completo de subida y bajada, además se observa que las revoluciones están por encima del valor teórico, esto puede ser debido a que en algunas ocasiones las olas producidas por el canal son un poco más grandes; en contraparte se observa que los escenarios $3,6,9$ y 12 fueron los que menos se aproximaron, esto puede ser debido a que estos tuvieron en común el periodo de ola de $2 \mathrm{~s}$, tiempo en el cual la boya no alcanzaba a terminar de bajar cuando se encontraba con la ola siguiente o en ocasiones la ola impactaba de manera directa a la boya sin causar el efecto de subida y bajada deseado.

TABla 3. Comparación de las ReVoluciones POR Minuto.

\begin{tabular}{|l|l|l|}
\hline Escenario & Revoluciones por minuto teóricas & Revoluciones por minuto del dispositivo \\
\hline 1 & 189 & 196 \\
2 & 265 & 156 \\
3 & 332 & 106 \\
4 & 158 & 169 \\
5 & 222 & 98 \\
6 & 277 & 67 \\
7 & 126 & 141 \\
8 & 177 & 130 \\
9 & 222 & 103 \\
10 & 94 & 75 \\
11 & 132 & 86 \\
12 & 165 & 80 \\
\hline
\end{tabular}

Fuente: Autores.

En la Tabla 4 se muestran los datos de voltaje y amperaje obtenidos a través del multímetro y el respectivo cálculo teórico y real del potencial producido en cada uno de los escenarios; además la eficiencia del equipo al comparar las potencias reales con las teóricas.

TABla 4. VALORES REPRESENTATIVOS DEL DISPOSITIVO DISEÑADO OBTENIDOS EN EL CANAL DE OLEAJE.

\begin{tabular}{|l|l|l|l|l|l|}
\hline Escenario & Voltaje $(\mathrm{V})$ & Amperaje $(\mathrm{mA})$ & Potencia teórica $(\mathrm{W})$ & Potencia real $(\mathrm{W})$ & Eficiencia $(\%)$ \\
\hline 1 & 10.32 & 125 & 154.35 & 5.16 & 3.34 \\
2 & 9.47 & 121.2 & 110.25 & 4.59 & 4.17 \\
3 & 8.5 & 114 & 88.20 & 3.87 & 4.40 \\
4 & 8.45 & 123.8 & 107.18 & 4.18 & 3.90 \\
5 & 9.3 & 117.2 & 76.56 & 4.35 & 5.69 \\
6 & 7.4 & 103.5 & 61.25 & 3.06 & 5.00 \\
7 & 10.7 & 120 & 68.60 & 5.13 & 7.49 \\
8 & 9.1 & 116.4 & 49.00 & 4.23 & 8.65 \\
9 & 9.7 & 115.9 & 39.20 & 4.49 & 11.47 \\
10 & 8.7 & 98 & 38.58 & 3.41 & 8.85 \\
11 & 9.3 & 104.6 & 27.56 & 3.89 & 14.15 \\
12 & 9.1 & 100.9 & 22.05 & 3.67 & 16.70 \\
\hline
\end{tabular}

Fuente: Autores.

Teniendo en cuenta que en la sección II, subsección B (Fuentes de datos de oleaje) se obtuvo como resultado que la altura y periodo promedio de ola para la zona costera del departamento de Córdoba es de $0.6 \mathrm{~m}$ y $6 \mathrm{~s}$ respectivamente, se puede calcular el potencial energético undimotriz teórico a través de (11) como sigue:

$$
\begin{aligned}
& P_{S}=0.49(0.6)^{2} \\
& P_{s}=1.0584\left[\frac{K W}{m}\right]=1058.4\left[\frac{W}{m}\right]
\end{aligned}
$$


Luego de realizar el análisis estadístico a los datos de potencia real obtenidos en la Tabla 4, se determinó (12), la cual es la ecuación corregida luego de aplicar la validación de supuestos; esta sirve para obtener una aproximación de la potencia del dispositivo con los valores de altura y periodo propios del departamento.

$$
y=1.8244+0.8151 T+0.8150 H
$$

Por lo tanto el potencial energético undimotriz obtenido a través del equipo diseñado en el presente trabajo, usando las condiciones de la zona costera del departamento de Córdoba es de 7.2 W. Este valor nos indica que es posible obtener energía Undimotriz en el departamento de Córdoba incluso con un equipo pequeño como el que se usó aquí, por lo tanto es viable la generación de energía Undimotriz en el departamento, ya que al mejorar la eficiencia de los dispositivos se puede aumentar considerablemente la cantidad de energía absorbida, debido a que el potencial teórico es de y mejorar la calidad de vida de las personas que viven con deficiencias de energía eléctrica en las zonas costeras.

\section{Discusión}

Al observar los datos de la Tabla 4, se aprecia que existe una gran diferencia entre la potencia real y la teórica, esto se puede deber a varios factores que influyentes en el diseño del dispositivo, entre ellos se mencionan los siguientes:

La ecuación (11) de potencial energético undimotriz teórico tiene unidades de esto quiere decir la cantidad de kiloWats presentes en un metro de frente de ola; como se observó en la sección 2, subsección B (Geometría y dimensiones de la boya), el diámetro de boya elegido para el presente trabajo es de $0.6 \mathrm{~m}$; así mismo en el momento de la validación del dispositivo en el laboratorio de hidráulica y mecánica de fluidos de la Universidad Nacional de Colombia se observó que la superficie de la boya en contacto con el agua era mucho más pequeña, al realizar los cálculos de flotabilidad con fluidos hidrodinámicos se encontró que la superficie real de la boya en contacto con el agua es aproximadamente de $0.164 \mathrm{~m}$; esto es un factor importante, dado que solo se está absorbiendo el 16.4\% del frente de ola.

Otro aspecto importante es la afirmación hecha por [47] según la cual un absorbedor puntual oscilando verticalmente puede reflejar menos del 50\% de la energía incidente; más aún, asegura que diversos estudios han demostrado que estos dispositivos en la práctica no son capaces de captar más de un $20 \%$ de la energía incidente, disminuyendo considerablemente la cantidad de energía que se puede absorber, esto último se confirma con la información descrita por [19], acerca del convertidor de energía undimotriz Sharp Eagle I (10 kW) el cual había estado funcionando cerca de Wanshan Island durante casi 6000 horas, con una eficiencia total de conversión de $16.76 \%$. En la Tabla 4 se observa que en todos los escenarios evaluados la eficiencia del equipo no supero el $16.70 \%$, todo esto refleja la problemática descrita por [48] acerca de la baja eficiencia de los dispositivos convertidores de energía undimotriz.

Otro elemento importante en el análisis comparativo de potencia teórica vs real del generador elegido, debido a que el PK244-02A-C46 de la marca VEXTA [49] utilizado en el dispositivo trabaja con un torque de entre 0.3 y $0.4 \mathrm{Nm}$ para producir voltaje; como el dispositivo produce en teoría un torque de $10 \mathrm{Nm}$ se puede inferir que el generador no aprovecha la totalidad de energía entregada por las olas. Sin embargo, esto era algo esperado de antemano ya que al momento de elegir el generador para el dispositivo se dialogó con expertos en electrónica, los cuales recomendaron que para estas situaciones en las cuales la entrada no se puede controlar, lo mejor sería diseñar un generador acorde a las necesidades, pero esto se salía de los objetivos del presente proyecto. Otra observación importante es como la eficiencia disminuye al aumentar la altura y periodo, esto se puede deber a que en los escenarios con bajas revoluciones presenta una baja altura y por lo tanto un torque pequeño, de esta manera el generador puede trabajar en condiciones cercanas a las establecidas en sus valores nominales, en consecuencia al aumentar la altura y periodo se empieza a desperdiciar torque. No obstante se observa que a medida que aumentan la altura y periodo de ola, la potencia obtenida aumenta. 
Un último aspecto importante por mencionar en el diseño del dispositivo es que la medición del voltaje y amperaje se hizo de manera visual en un multímetro, ya que esa fue la manera elegida por los autores para realizar las mediciones, esto pudo generar errores debido a la incertidumbre en el tiempo de toma de medidas; al momento de realizar las pruebas se evidenció que una forma más adecuada para medir dichos parámetros sería anexando un osciloscopio a la salida del generador, para poder definir de manera más clara los picos de las mediciones, así como el tiempo que toma en estabilizarse el sistema.

En la búsqueda bibliográfica realizada a nivel de Colombia [11], [24]-[27], se encontraron pocas investigaciones en el tema de energía undimotriz. Con respecto al potencial energético de la zona costera del departamento de Córdoba, el estudio más cercano fue el [32] realizado en Isla Fuerte, al comparar el resultado teórico obtenido en el presente artículo a través de la ecuación (11), el cual fue de $1058.4\left(\mathrm{Wm}^{-1}\right)$ y concuerda con el encontrado por [33], el cual concluye que existen aproximadamente $1\left(\mathrm{KWm}^{-1}\right)$. Se observó adicionalmente que los estudios realizados en el país se enfocan generalmente en mediciones indirectas del oleaje y no en el diseño de los dispositivos que puedan aprovechar el recurso, tal como se muestra en [28] o convertidores que aprovechen las condiciones reales de la zona costera Caribe.

$\mathrm{Al}$ analizar el resultado obtenido en (12) el cual muestra que se podrían aprovechar $7.2 \mathrm{~W}$ de potencia con las condiciones del departamento, se concluye que es posible aprovechar un porcentaje de la energía que contiene el océano en la zona costera de Córdoba, sin embargo el proyecto en este momento no es económicamente viable, pero es posible que a futuro contando con buenos recursos, mejorando el diseño e implementando elementos más adecuados se podría trabajar en un convertidor de energía undimotriz que aproveche por lo menos el $20 \%$ del potencial teórico existente en el departamento, es decir unos $200 \mathrm{~W}$. Esto para poder ofrecer un producto acorde a las características de la zona que genere una solución energética a las zonas de difícil acceso o no interconectadas a la red eléctrica nacional y así aprovechar el recurso undimotriz del departamento de Córdoba.

\section{Conclusiones}

Se demostró que existe un potencial undimotriz aprovechable en la zona costera del departamento de Córdoba y se calculó que es teóricamente de 1058.4 (Wm-1).

Se diseñó un dispositivo absorbedor puntual tipo on-shore capaz de transformar la energía potencial de las olas en electricidad, encontrando que la máxima potencia producida fue de $5.16 \mathrm{~W}$ con una altura de $0.3 \mathrm{~m}$ y $3.5 \mathrm{~s}$.

Para la geometría esférica elegida para la boya los periodos iguales o superiores a tres segundos presentaban un mejor aprovechamiento de la energía potencial de las olas, debido a que la boya alcanzaba a subir y bajar completamente con el pasar de las olas. Adicionalmente la geometría de la boya aun cuando se eligió por ser la que demostró un mejor comportamiento estructural, no es la apropiada para el dispositivo, debido a que su superficie no aprovecha completamente el frente de ola.

El potencial que produciría el dispositivo en la zona costera del departamento de Córdoba es de $7.204 \mathrm{~W}$ teniendo en cuenta una altura de $0.6 \mathrm{~m}$ y un periodo de $6 \mathrm{~s}$, los cuales serían suficientes para la carga de pequeños dispositivos electrónicos tales como celulares, tablets, gps o lectores electrónicos, etc.

\section{FinANCIAMIENTO}

El financiamiento de este proyecto fue dado por los mismos autores.

\section{REFERENCIAS}

[1] Instituto de Planificación y Promoción de Soluciones Energéticas para las Zonas No Interconectadas, Informe de Rendición Social de Cuentas, IPSE, Sep. 2015. [Online]. Available: http://www.ipse.gov.co/ atencionciudadano/seguimiento-spqr/rendicion-de-cuentas

[2] A. Pinilla, "Soluciones energéticas para zonas rurales (¿en el posconflicto?)", Rev. Ing., no. 44, pp. 36-39, Jun. 2016. https://doi.org/10.16924/riua.v0i44.921

[3] R. Peon, S. Leon \& D. Irvine-Halliday, "Solid state lighting for developing countries: A cost effective solution, facilitator of gender equity and encourager of peace" in Proceedings of 8th World Renewable Energy Congress, WREC/WREN, Denver, CL, USA, 2004. 
[4] C. Franco, I. Dyner \& S. Hoyos, "Contribución de la energía al desarrollo de comunidades aisladas no interconectadas: un caso de aplicación de la dinámica de sistemas y los medios de vida sostenibles en el suroccidente colombiano", Dyna, vol. 75, no. 154, pp. 199-214, Mar. 2008.

[5] M. Navarro, J. Hernández, E. Benedetti \& A. González, "Aprovechamiento de Energía Offshore: Avances y Perspectivas", Rev. ESAICA, vol. 2, no. 1, pp. 3-6, Ene. 2016. http://dx.doi.org/10.15649/24225126.421

[6] D. Vininanza, L. Margheritini, P. Kofoed \& M. Buccino, "The SSG Wave Energy Converter: Performance, Status and Recent Developments," Energies, vol 5, no. 12, pp. 193-226, Dic. 2012. [Online]. Available: https://www.researchgate.net/publication/233420375_The_SSG_Wave_Energy_Converter_Performance_Status_and_Recent_Developments

[7] A.F.d.O. Falcão, "Wave energy utilization: A review of the technologies," Renew. Sust. Energ. Rev, vol. 14, no. 3, pp. 899-918, Apr. 2010. [Online]. Available: https://www.sciencedirect.com/science/article/abs/ pii/S1364032109002652

[8] P. Ruiz, J. Villate \& F. Salcedo, "Wave energy: state of the art and future perspectives," Dyna, vol. 83, no. 3, pp. 139-147. Apr. 2008.

[9] R. Waters, "Energy from Ocean Waves: Full Scale Experimental Verification of a Wave Energy Converter”, PhD Dissertation, Fac. Sci. Tech., Uppsala Univ, Uppsala, SE, Dic. 2008.

[10] G. De Backer, "Hydrodynamic design optimization of wave energy converters consisting of heaving point absorbers", PhD Dissertation, Dept. CE, Ghent Univ, Ghent, BE, Dic. 2009.

[11] R. Torres \& C. Andrade, "Potencial en Colombia para el aprovechamiento de la energía no convencional de los océanos", Boletín Científico CIOH, vol. 24, pp. 11-25, Aug. 2006. https://doi.org/10.26640/22159045.145

[12] Comisión Colombiana del Océano, El océano: maravilla terrestre. Bogotá, D.C.: Publicaciones CCO, 2013.

[13] J. Cruz, Ocean wave energy: Current status and future perspectives. Berlin: Springer, 2008. https://doi. org/10.1007/978-3-540-74895-3

[14] F. Manzano, A. Alcayde, F. Montoya, A. Zapata \& C. Gil, "Scientific production of renewable energies worldwide: An overview," Renew. Sust. Energ, vol. 18, pp. 134-143, Feb. 2013. https://doi.org/10.1016/j. rser.2012.10.020

[15] D. Vicinanza, P. Contestabile \& V. Ferrante, "Wave energy potential in the north-west of Sardinia (Italy)," Renew. Energy, vol. 50, pp. 506-521, Feb. 2013. https://doi.org/10.1016/j.renene.2012.07.015

[16] G. Iglesias, M. López, R. Carballo, A. Castro, J. Fraguela \& P. Frigaard, "Wave energy potential in Galicia (NW Spain)," Renew. Energy, vol. 34, no. 11, pp. 2323-2333, Nov. 2009. https://doi.org/10.1016/j. renene.2009.03.030

[17] A. González, R. Ruiz, Q. Hernández, A. Fernández \& F. Manzano, "Wave energy resource assessment at southern coast of the Gulf of Mexico", Dyna, vol 82, no. 193, pp. 49-55, Sep. 2015. https://doi. org/10.15446/dyna.v82n193.45530

[18] R. Cáceres, "Energías renovables no convencionales: factibilidad de la energía oceánica en Chile", Tesis Grado, Fac. Econ. Neg., UCH, SCL, CL, 2015.

[19] Ocean Energy System, “Annual Report 2015,” OES. [Online]. Available: https://www.ocean-energy-systems.org/library/annual-reports/document/oes-annual-report-2015/

[20] P. Fernández, La energía del mar. Santander: Cantabria, ES: Universidad. de Cantabria, 2007. [Online]. Available: https://pfernandezdiez.es/es/libro/?id=5

[21] J. Fernández, "Una aproximación al aprovechamiento de la energía de las olas para generación de electricidad”, Tesis Grado, Dpto. Ing. Eléc., UPM, MAD, ES. 2008.

[22] P. Correa, "Estudio del comportamiento de un mecanismo de boyas para obtener energía undimotriz", Tesis Grado, Fac. Cienc. Fís. y Mat., UCH, SCL, CL, 2016

[23] J. Falnes, "A review of wave-energy extraction", Mar. Struct, vol. 20, no. 4, pp. 185-201, Oct. 2007. https://doi.org/10.1016/j.marstruc.2007.09.001

[24] M. Tovar \& L. Yamá, "Análisis de prefactibilidad para la generación de energía eléctrica mediante energía Undimotriz en Tumaco (Nariño)", Tesis Grado, Fac. de Ing., UniSalle, Bog., CO, 2009.

[25] S. Ortega, A. Osorio \& P. Agudelo, "Estimation of the wave power resource in the Caribbean Sea in areas with scarce instrumentation. Case study: Isla Fuerte, Colombia," Renew. Energy, vol. 57, pp. 240-248, Sep. 2013. https://doi.org/10.1016/j.renene.2012.11.038

[26] C. Appendini, C. Urbano, B. Figueroa, C. Dagua, A. Torres \& P. Salles, "Wave energy potential assessment in the Caribbean Low Level Jet using wave hindcast information," Appl. Energy, vol. 137, pp. 375-384, Jan. 2015.

[27] R. Jaramillo \& A. Garcés, "Generación Undimotriz: Integración con la red y análisis de resonancia subsíncrona,” Energética, vol. 45, pp. 49-59. Jan. 2015. https://doi.org/10.15446/energetica

[28] R. Borrás, R. Ferreiro, F. Miguélez \& C. Fernández, "Making Use of Coastal Wave Energy: A proposal to improve oscillating water column converters," Dyna, vol. 81, no. 185, pp. 211-218. Jun. 2014. https:// doi.org/10.15446/dyna.v81n185.38572

[29] J. Racero, J. Ballesteros \& J. Pérez, "Mamíferos del departamento de Córdoba-Colombia: historia y estado de conservación,” Biota Colombiana, vol. 16, no. 2, pp. 128-148, Jul. 2015.

[30] A. Marín, Estadísticas sobre el recurso agua en Colombia. Bogotá, D.C.: HIMAT, MinAgricultura, 1992.

[31] J. Martínez, "Propuesta metodológica para la estimación de la cota de inundación en la zona costera del caribe colombiano", Magister Thesis, Esc. Geocienc. MA, Fac. de Minas, UNAL, MDL, CO, 2010.

[32] S. Ortega, "Estudio de aprovechamiento de la energía del oleaje en Isla Fuerte (Caribe Colombiano)", Magister Thesis, Esc. Geocienc, Med. Amb., Fac. de Minas, UNAL, MDL, CO, 2010. 
[33] N. Booij, R. C. Ris \& L. H. Holthuijsen, "A third-generation wave model for coastal regions: 1. Model description and validation," JGR, vol. 104, no. C4, pp. 7649-7666, Apr. 1999. https://doi.org/10.1029/98JC02622

[34] CIOH, Sistema de Medición de Parámetros Oceanográficos y de Meteorología Marina SMPOMM, Bog, CO: DIMAR. [Online]. Available: https://www.dimar.mil.co/content/sistema-de-medicion-de-parametros-oceanograficos-y-de-meteorologia-marina-smpomm

[35] CIOH. Boletín mensual, Bog, CO: CECOLDO, [Online]. Available: http://www.cioh.org.co/meteorologia/ ResumenClimatologico.php

[36] P. Beirao \& M. dos Santos, "Design and analysis of buoy geometries for a wave energy converter," IJEEE, vol. 5, no. 2, p. 91, May. 2014. https://doi.org/10.1007/s40095-014-0091-7

[37] P. Beirão \& C. Malça, "Hydraulic Power Take-off and Buoy Geometries Charac-terisation for a Wave Energy Converter," EPE, vol. 5, no. 04, pp. 72-77, Jul. 2013. https://doi.org/10.4236/epe.2013.54B014

[38] J. Falnes, Ocean waves and oscillating systems: linear interactions including wave-energy extraction, Cambridge: Cambridge University Press, 2002. https://doi.org/10.1017/CBO9780511754630

[39] D. Montoya, "Modelado y control de centrales undimotrices. Aplicación a sistemas oscilantes y osciladores de columna de agua", Magister Thesis, US, Sevilla, ES, 2010.

[40] H. Sarlak, M. Seif \& M. Abbaspour, "Experimental investigation of offshore wave buoy performance," $J$. Mar. Eng. Technol, vol. 6, no. 11, pp. 1/E-11/E, Sep. 2010.

[41] C. Velez, Z. Qu, K. C. Lin \& S. Jin, "Design, Modeling and Optimization of an Ocean Wave Power Generation Buoy," Mar. Technol. Soc. J, vol. 48, no. 4, pp. 51-60, Jul. 2014. https://doi.org/10.4031/ MTSJ.48.4.6

[42] A. Biesheuvel \& S. Spoelstra, "The added mass coefficient of a dispersion of spherical gas bubbles in liquid," Int. J. Multiphas. Flow, vol. 15, no. 6, pp. 911-924, Nov. 1989. https://doi.org/10.1016/03019322(89)90020-7

[43] K. H. Vu, B. Chenu \& K. P. Thiagarajan, "Hydrodynamic damping due to porous plates", in Proceedings of the WSEAS International Conference on Fluid Mechanics, WSEAS, Corfu Island, GR, pp. 17-19, Aug. 2004.

[44] Z. Demirbilek \& C. Vincent, Water Wave Mechanics Coastal Engineering Manual, EM 1110-2-1100, USACE, Washington, USA. 2002.

[45] J. Sá, A. Sarmento, F. Gardner, P. Beirão \& A. Brito-Melo, "Time domain model of the Archimedes Wave Swing wave energy converter" in Proceedings of the Sixth European Wave and Tidal Energy Conference, EWTEC, Glasgow, UK, pp. 91-97, Aug. 2005.

[46] C. Carleos \& N. Corral, "Paquetes estadísticos con licencia libre (I)", REMA, vol. 18, no. 2, pp. 12-37, 2013.

[47] R. Rodríguez, P. Ricci, A. Marón, M. E. Prieto, D. Fernández \& M. Taboada, "Diseño y ensayos del sistema de fondeo de un convertidor de energía de las olas", CEHIPAR, vol. 209, pp. 1-31, 2008.

[48] B. Drew, A. Plummer \& M. Sahinkaya, "A review of wave energy converter technology. Proceedings of the Institution of Mechanical Engineers, Part A," JPEE, vol. 223, no. 8, pp. 887-902, Dec. 2009. https:// doi.org/10.1243/09576509JPE782

[49] "Stepping Motors (Motor Only)" Oriental Motors, Elk Grove Village, IL, USA. [Online]. Available: http:// www.orientalmotor.com/products/pdfs/2012-2013/A/usa_st_pk_motor_only_overview.pdf

[L1] República de Colombia. Congreso de la República. Ley 1715. (2014, may. 13). Energías renovables no Convensionales. Diario Oficial: No. 49.150.

[L2] República de Colombia. Presidencia de la República. Decreto 2143. (2015, nov. 4). Decreto Único Reglamentario del Sector Administrativo de Minas y Energía. Diario Oficial: No. 49.686.

Yair Gonzalez Doria recibió el título en Ingeniería mecánica de la Universidad Pontificia Bolivariana (Colombia) y el título de maestría en Administración industrial de la Universidad del Norte (Colombia). Sus intereses principales de investigación incluyen la simulación. https://orcid.org/0000-0002-4468-6134

Manuel Herrrera Medina recibió título de Ingeniería mecánica en 2017. Sus intereses en investigación incluyen diseño mecánico y simulación.

César Ávila Diaz recibió el título de Ingeniería mecánica en 2017. Sus intereses en investigación incluyen diseño mecánico y simulación. 